\title{
Qualification of multi-fiber interconnect solutions for payload data processing
}

Cathy Combet, Houda Brahimi, Jean-Baptiste Sauveplane

Cathy Combet, Houda Brahimi, Jean-Baptiste Sauveplane, "Qualification of multi-fiber interconnect solutions for payload data processing," Proc. SPIE 11852, International Conference on Space Optics - ICSO 2020, 1185253 (11 June 2021); doi: 10.1117/12.2599924

SPIE Event: International Conference on Space Optics - ICSO 2021, 2021, Online Only 


\section{International Conference on Space Optics-ICSO 2020}

Virtual Conference

30 March-2 April 2021

Edited by Bruno Cugny, Zoran Sodnik, and Nikos Karafolas
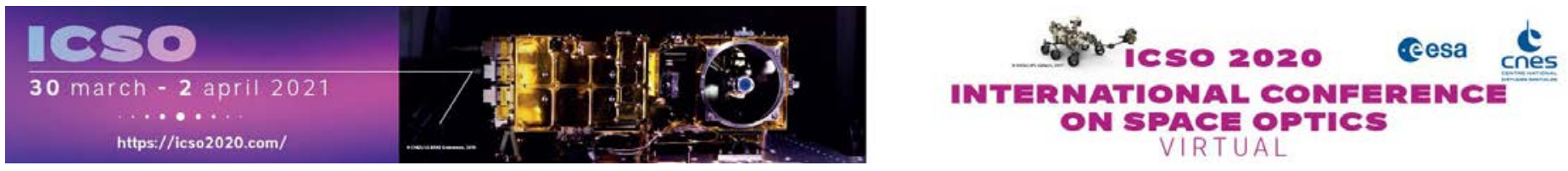

\section{Qualification of multi-fiber interconnect solutions for payload data processing}

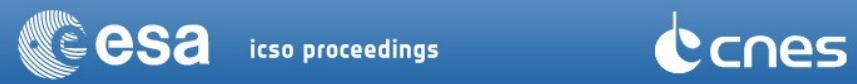




\title{
Qualification of Multi-fiber Interconnect Solutions for Payload Data Processing
}

\author{
Cathy Combet ${ }^{\mathrm{a}}$, Houda Brahimi ${ }^{\mathrm{b}}$, Jean-Baptiste Sauveplane ${ }^{\mathrm{c}}$ \\ ${ }^{a}$ Radiall, 15 rue Garenne, 38070 Saint Quentin Fallavier, France; \\ ${ }^{\mathrm{b}}$ Thales Alenia Space, 26 Av Jean-François Champollion B.P.33787 31037 Toulouse Cedex1, \\ France ; \\ ${ }^{\mathrm{c}} \mathrm{CNES}, 18$ Avenue Edouard Belin, 31400 Toulouse, France
}

\begin{abstract}
It is essential to use multi-fiber interconnect solutions to meet requirements for bandwidth and the amount of data needed to transmit in payload applications without compromising weight and performance.

In order to have multi-fiber solutions available and qualified for space applications in severe environments, CNES, Thales Alenia Space (TAS) and Radiall have specified, designed and qualified a full optical link based on a high-density, 12-fiber MT ferrule. The methodology to qualify this entire solution duplicates the real conditions of the product in its operating conditions. The qualification test plan includes mechanical, thermal, outgassing and radiation tests and has been successfully completed. The sequence of tests has been properly defined in accordance with state-of-the-art technology and the expected performance in severe environments. One test file with mating, vibration, shock and temperature cycling and one test file with mating, vibration, shock and temperature storage were conducted to guarantee that the optical and mechanical properties are maintained after the test sequence.

The full optical link consists of harnesses, combining ruggedized optical contacts (Q-MTitan ${ }^{\mathrm{TM}}$ ) with 12 radiation resistant fibers. These assemblies are connected by an 8 or 10-cavity multipin panel feedthrough disconnect connector.

The Q-MTitan ${ }^{\mathrm{TM}}$ optical contact was designed for use in size 8 Quadrax cavities of off-the-shelf multipin connectors used in the defense and aerospace markets, such as MIL-DTL-38999, ARINC 600, EPX EN4644 and EN4165. This mature contact has demonstrated its performance and value as the baseline for ARINC 846, the standard of the aerospace industry. It features a compact, lightweight and ruggedized body that incorporates and protects the MT ferrule to guarantee the best optical performance in the most demanding environments. The contact can be terminated on round cable or ribbon fibers without needing specific accessories that would have increased the overall weight.

The multipin connector design was driven by the search for the best tradeoff in terms of density, cost and performance, such as mechanical, thermal and radiation resistance.

The harnesses built with these interconnect solutions and the use of Radiation-Hardened fibers (RadHard) fibers and cables have been tested and qualified following the test plan established in collaboration with CNES and TAS to be as close as possible to the operating application.
\end{abstract}

In this paper, we describe the complete optical solution, its qualification test plan and results that demonstrate the possibility of using multi-fiber interconnect solutions inside and between equipment of payload applications.

Keywords: Multi-fiber connector, optical, interconnect, qualification test plan, qualification results, Q-MTitan ${ }^{\mathrm{TM}}$, HDQX, connector

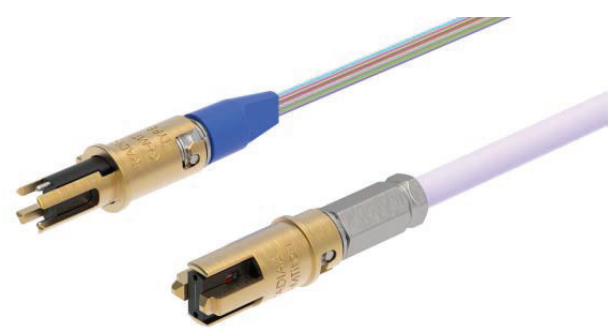

Figure 1. Q-MTitan ${ }^{\mathrm{TM}}$ 12-fiber contact 


\section{INTRODUCTION}

In order to have multi-fiber solutions available and qualified for severe environments of space applications, CNES, Thales Alenia Space (TAS) and Radiall have specified, designed and qualified a full optical link based on a high-density, 12-fiber MT ferrule. In this paper, we describe the complete optical solution, its qualification test plan and results that demonstrate the possibility of using multi-fiber interconnect solutions inside and between equipment of payload applications.

\subsection{Need for Multi-fiber}

New satellites must meet the demand for flexibility and configurability requested by telecommunication operators. The increase in bandwidth and number of RF access implies that the Digital Transparent Processor (DTP) is able to route, after digitization, a large amount of data, which is only possible using optical links. In 2017, CNES, TAS and Radiall started a study to specify, design and test multi-fiber interconnects solutions for space environment to address those applications requiring higher bandwidth in satellite communication (VHTS).

As a solution, Radiall designs and delivers optical links that meet the desired performance of space environment, such as density, ease of installation, weight, optical performance and resistance to radiation.

\subsection{Identification of the solutions}

Radiall Q-MTitan ${ }^{\mathrm{TM}}$ was identified as the high-density and ruggedized optical contact solution to withstand the space environments. The Q-MTitan ${ }^{\mathrm{TM}}$ contact can be terminated on round cable or ribbon fibers without needing specific accessories that would have increased the overall weight of the solution.

For this application, a multipin connector with 8 and 10 cavities was required. The HDQX 8 and HDQX 10 connectors offer a secure, reliable and size optimized solution to connect 96 to 120 fibers.

\subsection{Qualification process}

The methodology to qualify the entire solution has been defined to duplicate the real conditions of the product in its operating conditions. The Device Under Test (DUT) has been properly designed to duplicate the most stringent fiber arrangement. A particular attention was pointed on the bending radius and fixation points on the test vehicle. Two DUTs have been defined with representative tests sequences.

\section{DESCRIPTION OF THE OPTICAL SOLUTION}

\subsection{The complete optical link}

The full optical link consists of harnesses, combining ruggedized optical contacts (Q-MTitan ${ }^{\mathrm{TM}}$ ) with 12 radiation resistant fibers. These assemblies are connected by an 8 or 10 -cavity multipin panel feedthrough disconnect connector, called HDQX.

Both the contact and connector have been golden plated to increase the EMI performance as specified by the application. The connection offers SE higher than $55 \mathrm{~dB}$ up to $18 \mathrm{GHz}$.

The use of low outgassing materials upon ECSS-Q-ST-70-02C was mandatory. 


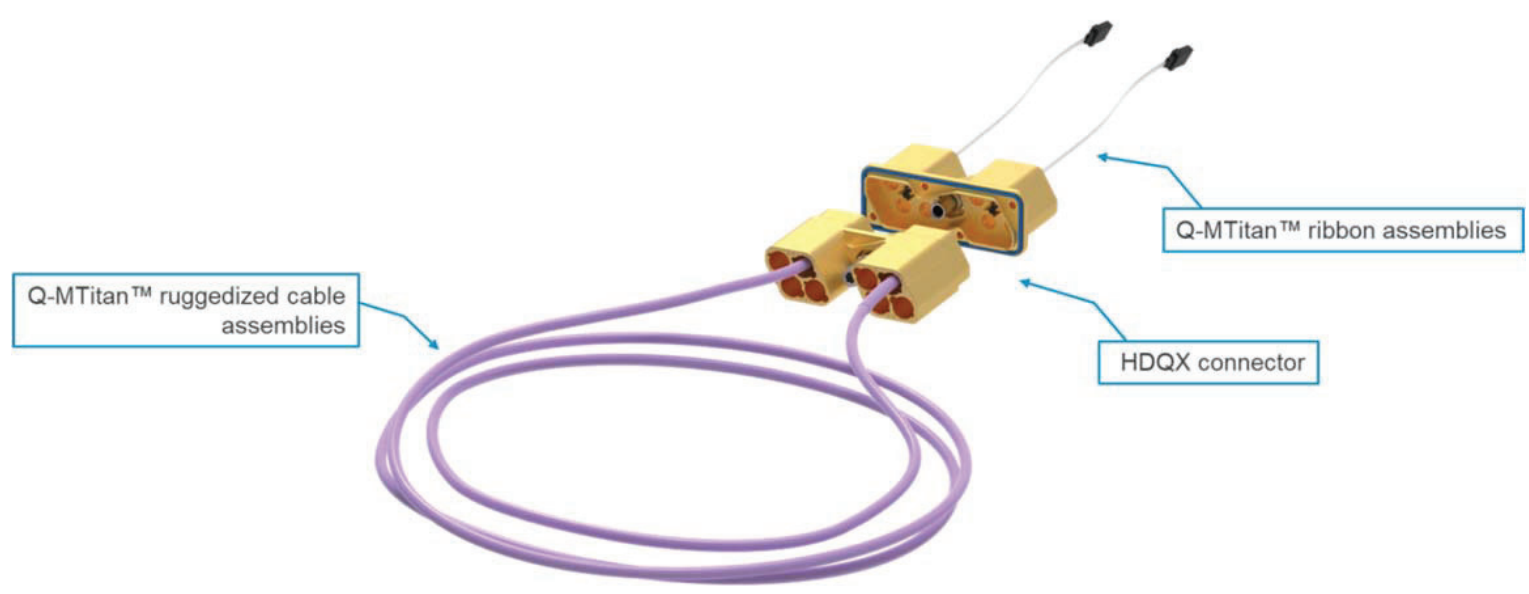

Figure 2. Complete Optical link with Q-MTitan ${ }^{\mathrm{TM}}$ cable assemblies and multi-cavity connector

\subsection{Q-MTitan ${ }^{\mathrm{TM}}$ multi-fiber contact}

The Q-MTitan ${ }^{\mathrm{TM}}$ optical contact was designed for high density, high data rate applications and can be used in existing size 8 Quadrax cavities of off-the-shelf multipin connectors that are available in the defense and aerospace markets, such as MIL-DTL-38999, Quick Fusio, NSX ARINC 600, EPX EN4644, and HDQX.

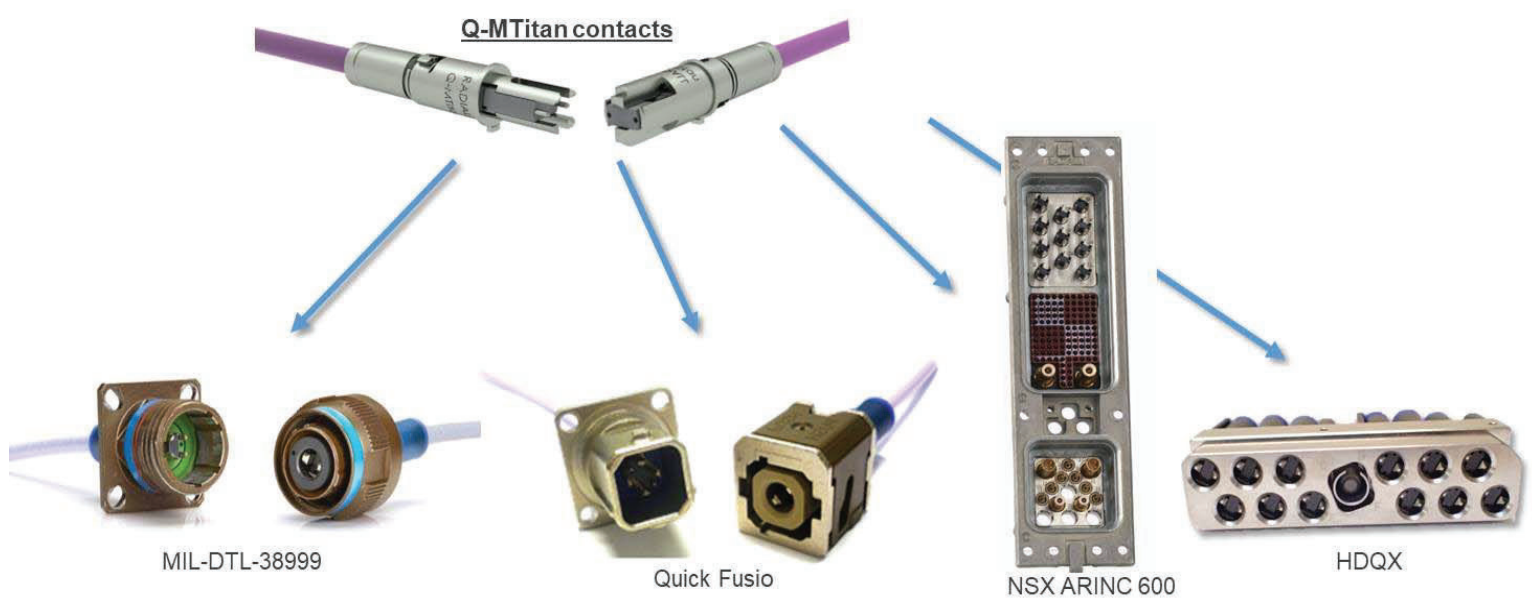

Figure 3. Q-MTitan ${ }^{\mathrm{TM}}$ fits various interconnect solutions

This mature contact has demonstrated its performance and value as the baseline for ARINC 846: the standard of the aerospace industry. It features a compact, lightweight and ruggedized body that incorporates and protects the MT ferrule to guarantee the best optical performance in the most demanding environments. Fielded with ribbon fibers or round multi-fiber cables, such as ARINC 802 (annex K), Q-MTitan ${ }^{\mathrm{TM}}$ offers a dense, robust and cost-effective solution per optical channel.

It also provides a simplified use by customers, and is further supported by through installation/maintenance/cleaning kits and fiber management solution to ease its integration. 


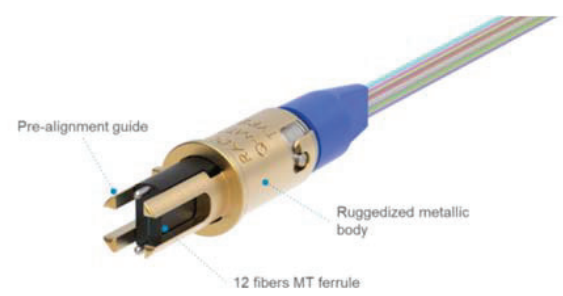

Figure 4. Q-MTitan ${ }^{\mathrm{TM}}$ ARINC 846 Aerospace industry standard for multi-fiber contact

\subsection{Multi-fiber cables}

Radiall terminates Q-MTitan ${ }^{\mathrm{TM}}$ on round cable and ribbon fibers.

The optical link to be qualified includes 2 types of cable assemblies to accommodate 2 different payload environments:

- Q-MTitan ${ }^{\mathrm{TM}}$ ruggedized cable assemblies - Length: $5 \mathrm{~m}$ :

Round cable made of a PFA external jacket, some aramid strength members and a PTFE internal tube.

These ruggedized cable assemblies are fitted with 12 Super RadHard fibers: OM2 NON bend insensitive (Radius of curvature: $40 \mathrm{~mm}$ )

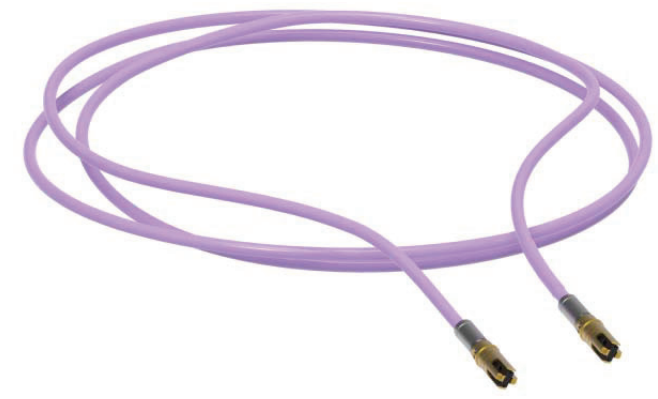

Figure 5. Q-MTitan ${ }^{\mathrm{TM}}$ to Q-MTitan ${ }^{\mathrm{TM}}$ cable assembly

- Q-MTitan ${ }^{\mathrm{TM}}$ ribbon assemblies - Length: $0.4 \mathrm{~m}$ :

Tthe ribbon is composed of 12 RadHard fibers.

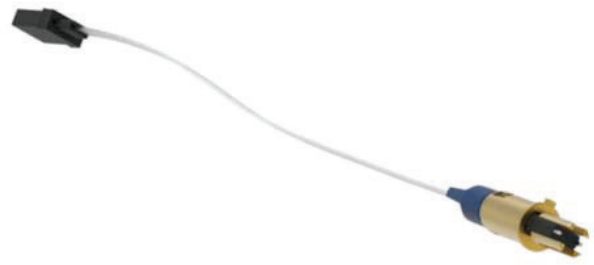

Figure 6. Q-MTitan ${ }^{\mathrm{TM}}$ to MT ferrule ribbon assembly

\subsection{Interconnect solution}

The multipin connector design was driven by the search for the best tradeoff in terms of density, weight, cost and performance, such as mechanical, thermal, shielding effect and radiation resistance.

Two versions of the connectors have been designed for the application: 1 version with 8 cavities for 8 Q-MTitan ${ }^{\mathrm{TM}}$ and 1 version with 10 cavities for 10 Q-MTitan ${ }^{\mathrm{TM}}$.

Both versions use a quick and easy-to-use locking mechanism with a screw system that secures the connection. 


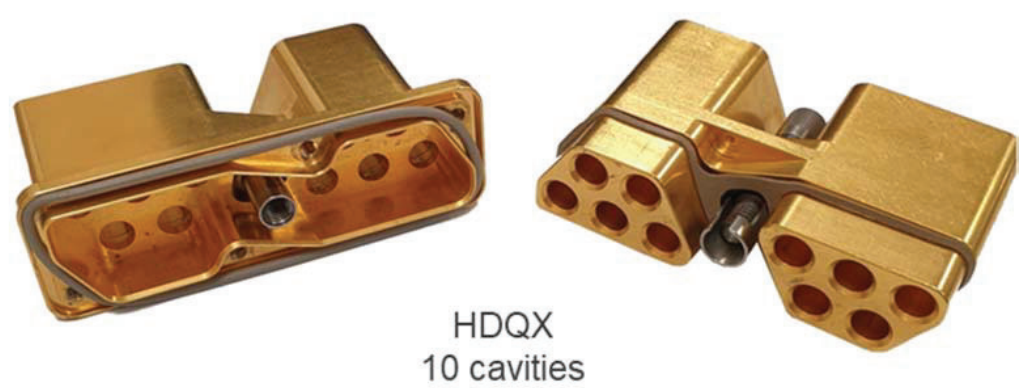

Figure 7. Multi-cavity connector HDQX

\section{QUALIFICATION TEST PLAN}

\subsection{Objectives}

The goal was to qualify the whole optical link in conditions that duplicate the real operating conditions of the products. A test plan has then been established in collaboration with CNES and TAS to be as close as possible to the operating conditions.

The qualification test plan includes mechanical, thermal, outgassing and radiation tests. The sequence of tests has been properly defined in accordance with state-of-the-art technology and the expected performance in severe environments. One test file with mating, vibration, shock and temperature cycling and one test file with mating, vibration, shock and temperature storage were conducted to guarantee that the optical and mechanical properties are maintained after the test sequence.

\subsection{Test plan}

Two files of tests with 2 DUTs have been defined. Visual inspection and optical measurements were performed at the beginning, after each test and at the end of the sequence. A Destructive Product Analysis (DPA) was performed on some samples at the end of the test files to check the contact retention force has not been deteriorated by the severe mechanical and environmental tests.

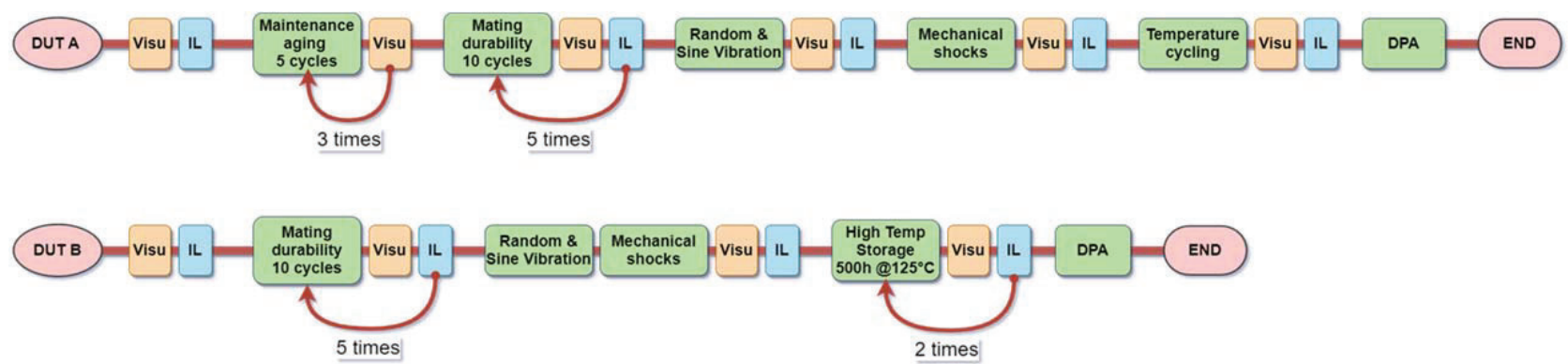

Figure 8. Test plan with 2 files of tests

Optical Sanctions:

- Evaluation@850 nm with overfilled launch conditions

- $\quad$ Q-MTitan ${ }^{\mathrm{TM}}$ IL Initial $<0.5 \mathrm{~dB}$

- IL variation after test $<1 \mathrm{~dB}$ (EOL: End of Life) for the full optical link

- $\mathrm{RL}<-20 \mathrm{~dB}$ 


\subsection{Devices Under Test (DUT)}

The connectors, contacts and cables used for qualification are in accordance with the design, construction and physical dimensions specified on the applicable product drawings. The contacts are selected at random from standard production.

The samples are distributed among 2 groups: DUT A with HDQX 8 and DUT B with HDQX 10. Both connectors are fully populated with Q-MTitan ${ }^{\mathrm{TM}}$ contacts. Each DUT is mounted on a dedicated plate with a defined routing and various attachment points to simulate the end-use condition of the assemblies.

A sample not subject to environmental test is monitored for reference.

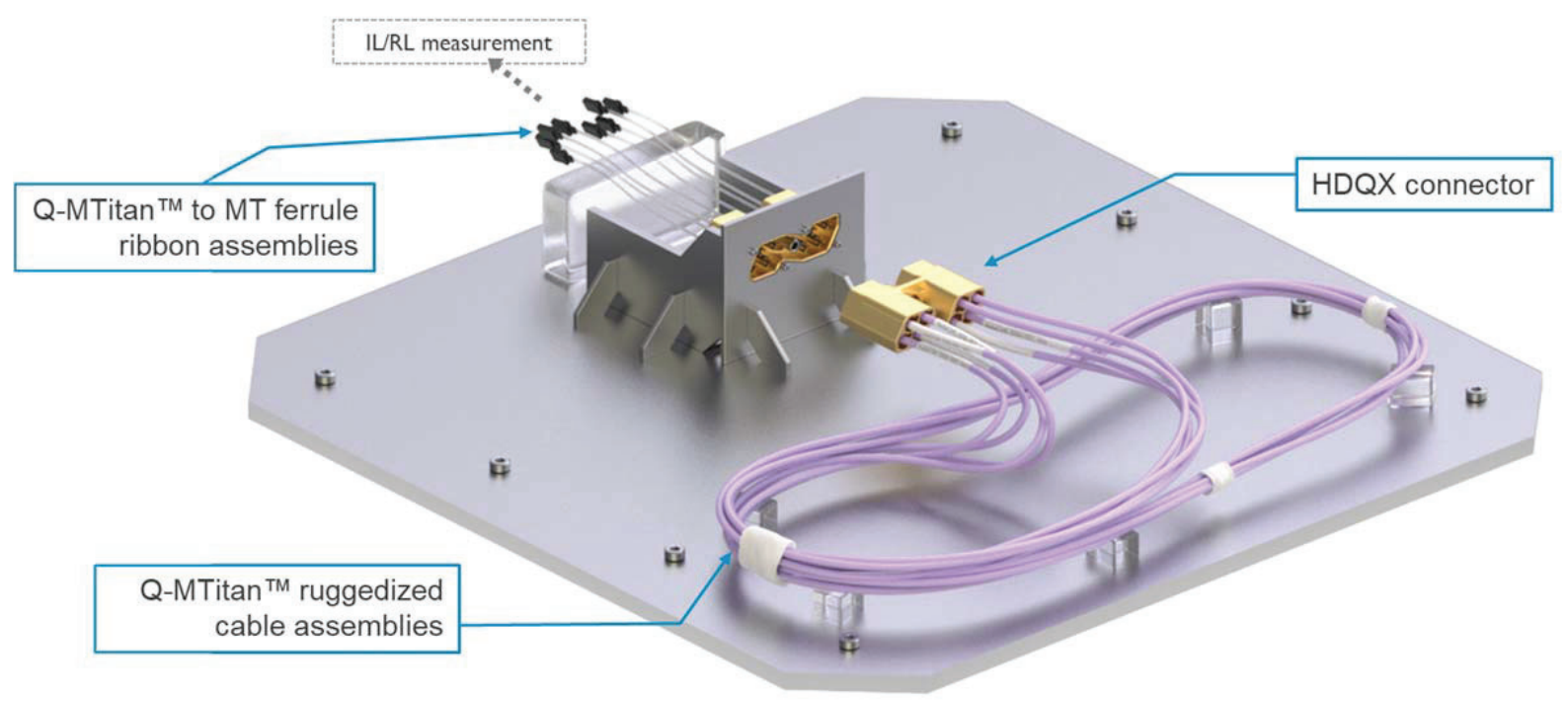

Figure 9. DUT schematic

\section{RESULTS OF THE QUALIFICATION}

\subsection{Summary of the results}

The test plan has been executed on both DUTs following the defined sequences of figure 6 . All the tests have passed the criteria. The qualification is completed and considered successful and the use of multi-fiber interconnect in space environment is demonstrated.

The results are summarized in figure 10 and the details of the individual tests are described in the following sections. 


\begin{tabular}{|c|c|c|c|c|}
\hline \multirow{2}{*}{ Test } & \multirow{2}{*}{ Standard } & \multirow{2}{*}{ Condition \& Sanctions } & \multicolumn{2}{|c|}{ DUT } \\
\hline & & & A & B \\
\hline Radiation & & On optical fibers & \multicolumn{2}{|c|}{$\boldsymbol{\nu}^{(1)}$} \\
\hline Visual Inspection & EN259I-101 & @ $\times 200$ : No damage, no contamination & $\checkmark$ & $\checkmark$ \\
\hline Insertion Loss IL & IEC 6|300-3-4 (B) & IL variation after test: $<\mid \mathrm{dB}$ for a full line (I Q-MTitan ${ }^{\mathrm{TM}}: 0.5 \mathrm{~dB}$ ) & $\checkmark$ & $\checkmark$ \\
\hline Return Loss RL & IEC $61300-3-6$ & $\mathrm{RL}<-20 \mathrm{~dB}$ & $\checkmark$ & $\checkmark$ \\
\hline Maintenance aging & & $\begin{array}{l}15 \text { cycles: insert/extract Q-MTitan }{ }^{T M} \text { on both sides of the connection while } \\
\text { unmated }\end{array}$ & $\checkmark$ & \\
\hline Mating durability & IEC $61300-2-2(A)$ & 50 cycles: Measurement every 10 cycles with cleaning authorized & $\checkmark$ & $\checkmark$ \\
\hline Random Vibration & & $40 \mathrm{G}$ rms up to $2,000 \mathrm{~Hz} ; 3$ min per axis on 3 axes & $\checkmark$ & $\checkmark$ \\
\hline Sine Vibration & & Level $20 \mathrm{G}$ up to $100 \mathrm{~Hz}$; I cycle in each of the 3 axes & $\checkmark$ & $\checkmark$ \\
\hline Mechanical shocks & & $2,000 \mathrm{G} ; 10,000 \mathrm{~Hz}$ & $\checkmark$ & $\checkmark$ \\
\hline Temperature cycling & IEC $61300-2-22$ & $-55 /+125^{\circ} \mathrm{C}, 50$ cycles, $5^{\circ} \mathrm{C} / \mathrm{min}$, dwell time: I h & $\checkmark$ & \\
\hline High Temperature storage & IEC $61300-2-18$ & I,000h@125C & & $\checkmark$ \\
\hline DPA: contact retention & IEC $61300-2-4$ & Retention force: $68 \mathrm{~N}$ for I min, then up to breakage & $v$ & $\checkmark$ \\
\hline Damp heat & IEC $61300-2-46$ & $+25^{\circ} \mathrm{C} /+55^{\circ} \mathrm{C}$, dwell time: $9 \mathrm{~h}$, number of cycles: $6,10^{\circ} \mathrm{C} / \mathrm{h}-\mathrm{RH}: 95 \%$ (on ribbon) & \multicolumn{2}{|c|}{$\boldsymbol{\nu}^{(1)}$} \\
\hline
\end{tabular}

Figure 10. Summary of the qualification results

\subsection{Maintenance aging results}

The test consists of repeating several insertions and extractions of the Q-MTitan ${ }^{\mathrm{TM}}$ contacts inside and from their cavities. A total of 15 cycles: insertion/extraction of Q-MTitan ${ }^{\mathrm{TM}}$ on both sides of the connection while unmated have been done. To insert and extract the Q-MTitan ${ }^{\mathrm{TM}}$ contact from its cavity, the operator follows Radiall's procedure and recommendations:

- Extraction is done with standard extraction tool (size 8 quadrax extraction tool)

- Insertion of Q-MTitan ${ }^{\mathrm{TM}}$ ruggedized cable assemblies is done without tool

- Insertion of Q-MTitan ${ }^{\mathrm{TM}}$ Ribbons assemblies is done using dedicated tool

The sanction for this test is the visual inspection. The test has passed the criteria and is considered successful.

\subsection{Mating durability results}

The test consists of repeating 50 cycles of mating/unmating the HDQX connectors fully populated. Cleaning is authorized when necessary.

The sanctions for this test are the visual inspection and Insertion Loss measurement. The test has passed the criteria and is considered successful.

\subsection{Vibration and shocks results}

The test consists of submitting the DUTs to high levels of vibrations and shocks. The DUTs are placed on a table with a mechanical impactor. The position of the pilot is closed to the connector. Three types of tests have been performed:

Random Vibration:

○ 40 Grms

○ Up to $2,000 \mathrm{~Hz}$ 
○ 3 min per axis on 3 axes

Sine Vibration:
○ $20 \mathrm{G}$
- Up to $100 \mathrm{~Hz}$
- 1 cycle in each axis on 3 axes

Shocks:

○ $\quad \mathbf{2 , 0 0 0 ~ G ~} 10,000 \mathrm{~Hz}$

The sanctions for these tests are the visual inspection and Insertion Loss measurement. The tests have passed the criteria and are considered successful.

\subsection{Temperature test results}

The tests consist of submitting the DUTs to high environmental conditions. The DUTs are placed in a dedicated climatic chamber. Two types of tests have been performed:

Temperature cycling:

○ 50 cycles: $\mathbf{- 5 5} /+\mathbf{1 2 5}^{\circ} \mathbf{C}, 5^{\circ} \mathrm{C} / \mathrm{min}, 1 \mathrm{~h}$ dwell time

High Temperature storage:

○1,000h@125ㄷ

The sanctions for these tests are the visual inspection and Insertion Loss measurement. The tests have passed the criteria and are considered successful.

\subsection{Visual inspection results}

A deep analysis under microscope has been performed after each test with the main sanction of no crack, no break, no fiber break, and no damage on the end faces.

All visual inspections were conforming to the criteria.

\subsection{Complementary test results}

\section{Damp heat:}

The test has been performed on ribbon assemblies with a temperature cycling of $+25^{\circ} \mathrm{C} /+55^{\circ} \mathrm{C} @ 95 \% \mathrm{HR}$. The temperature gradient was $10^{\circ} \mathrm{C} / \mathrm{h}$, and 6 cycles (dwell time: 9 hours) have been performed.

The sanctions for this test are the visual inspection and Insertion Loss measurement. The test has passed the criteria and is considered successful.

EMI:

Both the Q-MTitan ${ }^{\mathrm{TM}}$ contact and HDQX connector have been golden plated to offer EMI performance as required by the application. The connection offers SE higher than $55 \mathrm{~dB}$ up to $18 \mathrm{GHz}$.

Radiation:

Q-MTitan ${ }^{\mathrm{TM}}$ ruggedized cable assemblies are fitted with 12 Super RadHard fibers. These fibers feature the performance as represented in figure 11. (@ 0-15 MRad RIA: 0,12 dB). The PFA external jacket also keeps its integrity and doesn't break after irradiation. 


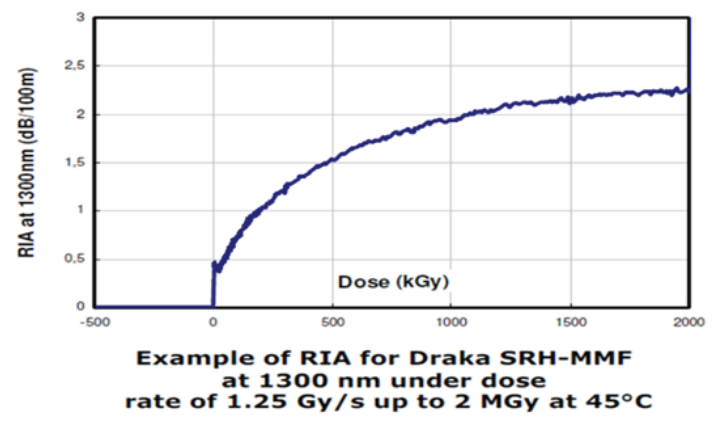

Figure 11. RIA of Super RadHard fibers

Q-MTitan ${ }^{\mathrm{TM}}$ ribbon assemblies are made of 12 RadHard fibers that feature the performance as described in figure 12. (@1MRad RIA: 0.07dB)

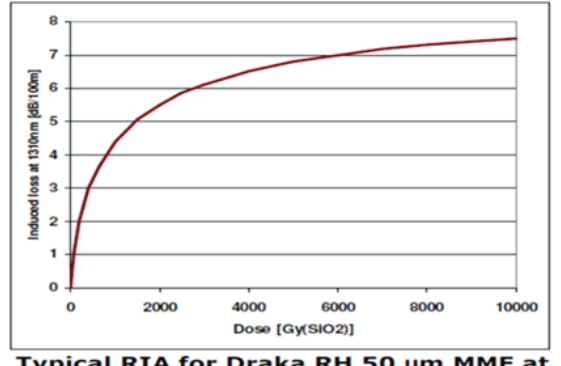

Typical RIA for Draka RH $50 \mu m$ MMF at

Figure 12. RIA of RadHard fibers

\section{CONCLUSION}

The optical backbone has successfully passed the qualification for space applications following the defined sequences and requirements.

All of the tests pass the environmental and mechanical testing campaigns, enabling the use of multi-fiber Q-MTitan ${ }^{\mathrm{TM}}$ optical interconnect in payload applications, such as the highly-demanding new generation of on-board digital processors. The optical harnesses have been subject to severe test files, including mating, vibration, shock, temperature cycling and temperature storage, to guarantee that the optical and mechanical properties are maintained after the test sequences.

As a result, Q-MTitan ${ }^{\mathrm{TM}}$ interconnect series is steadily available to be used for the new generation of Digital Transparent Processor (DTP) to deliver Very High Throughput Satellite (VHTS) connectivity.

This opens the road to more and more applications for fiber optics in space environments and justifies new developments for even higher density solutions with 24-fiber Q-MTitan ${ }^{\mathrm{TM}}$ interconnect solutions. 